KA-THEP-1989-6

Manusk. Fak. Math. u. Inf.

Mannheim Nr. 97-1989

\title{
Krichever-Novikov Algebras \\ for More than Two Points
}

\author{
Martin Schlichenmaier
}

97-1989

April 89

Faculty of Mathematics

and Computer Science

University of Mannheim, A5

D-6800 Mannheim 1

Federal Republic of Germany

\section{Abstract}

Krichever-Novikov algebras of meromorphic vector fields with more than two poles on higher genus Riemann surfaces are introduced. The structure of these algebras and their induced modules of forms of weight $\lambda$ is studied. 


\section{Introduction}

In the study of conformal field theory a recent generalization of the Virasoro Algebra was given by Krichever-Novikov [1][?] for Riemann surfaces of higher genus. Let $X$ be a fixed Riemann surface of genus $g$. We choose two "generic" points $P_{+}$and $P_{-}$ and consider the Lie algebra of meromorphic vector fields on $X$ which are holomorphic on $X \backslash\left\{P_{+}, P_{-}\right\}$. Krichever and Novikov showed that this algebra admits a central extension. They constructed a series of representation coming from the operation of the vector fields on the meromorphic forms of weight $\lambda$ which are holomorphic on

$X \backslash\left\{P_{+}, P_{-}\right\}$. This algebra (with or without central extension) is now usually called Krichever-Novikov algebra (short: KN algebra). For $g=0$, hence $X=\mathbb{P}^{1}$ (all varieties are over the complex numbers $\mathbb{C}$ ) this algebra is exactly the Virasoro algebra. A crucial part in the theory is the fixing of a distinguished basis of the vector fields and of the forms, the so called $\mathrm{KN}$ basis.

There is a meromorphic (1-) differential $\rho$ holomorphic on $X \backslash\left\{P_{+}, P_{-}\right\}$with residue +1 at $P_{+}$and residue -1 at $P_{-}$and only imaginary periods (see $\left.[3, p 116]\right)$. It was pointed out [2][6] that in string theory the level lines of the function $(Q$ denotes a point different from $P_{+}$and $P_{-}$)

$$
u(p)=\operatorname{Re} \int_{Q}^{P} \rho
$$

on $X \backslash\left\{P_{+}, P_{-}\right\}$could be interpreted as closed string configuration at the proper time $\tau$. Here $\tau$ gives the value of the function $u$ along the level line. As $\tau \rightarrow \pm \infty$ the level lines become circles around the points $P_{\mp}$ representing free incoming and outgoing strings.

Hence it is quite natural to ask for more strings coming together and interacting. In terms of $\mathrm{KN}$ algebras this is the question of the structure of the algebra of meromorphic vector fields which are holomorphic except at a certain points.

The aim of this paper is to give the results of such a generalization. Details of the calculation and further developments will be found in a separate publication [4]. For the generalities on Riemann surfaces, the theorem of Riemann-Roch and its application to the KN algebra for two points see [3]. For the interpretation in physics see [6-14].

\section{Fundamental definitions}

Let $X$ be a Riemann surface of genus $g$. We always assume $X$ to be compact and without boundary. $X$ can also be considered as a connected, projective, nonsingular curve over C. A formal sum of points $Q_{1}, \ldots, Q_{l} \in X$ with integer multiplicities

$$
D=\sum_{i=1}^{1} n_{i} Q_{i}
$$


is called a divisor. $K$ denotes the canonical divisor, resp. the canonical divisor class, resp. the canonical line bundle (which is the same as the holomorphic cotangent bundle). The sections of the canonical line bundle are the differentials. $K^{\lambda}:=K^{\otimes \lambda}$ is $\lambda$ times the tensor product of the bundle $K$ with itself. For $\lambda<0$ this means $K^{\lambda}:=\left(K^{*}\right)^{\otimes|\lambda|} . K^{*}$ denotes the dual bundle, the holomorphic tangent bundle. If we use the divisor notation, then $K^{\lambda}$ corresponds to the divisor $\lambda \cdot K$.

The definition of $K^{\lambda}$ with $\lambda \in \mathbf{Z}$ is clear. To define it for $\lambda$ half integer we have to choose a "square root" of $K$. This is not unique for $g \geq 1$. For arbitrary $\lambda$ we have to consider coverings of $X$. In this paper we will restrict ourselves to integer valued $\lambda$. The case of arbitrary $\lambda$ will be covered in [4].

The (meromorphic) sections of $K^{\lambda}$ are called (meromorphic) forms of weight $\lambda$. Forms of weight -1 are the vector fields. Let $D$ be a divisor as above then $H^{0}(X, \lambda K+D)$ is the vector space of meromorphic $\lambda$ - forms which are holomorphic outside the support of $D$ ( which is the set of points appearing with nonvanishing multiplicities) and have a zero of at least order $-n_{i}$ at the point $Q_{i}$. As usual a zero of negative order $m_{i}$ is interpreted as a pole of order $-m_{i}$. We use the notation ord $Q_{1}(8)$ for the order of the zero of the section at the point $Q_{i}$.

By the theorem of Riemann-Roch (see $[3, p .107])$ and using $\operatorname{deg}(\lambda K)=2 \lambda(g-1)$ we get

$$
\begin{aligned}
\operatorname{dim} H^{0}(X, \lambda K+D)-\operatorname{dim} H^{0}(X,(1-\lambda) K-D) & =\operatorname{deg}(\lambda K+D)-g+1 \\
& =(2 \lambda-1)(g-1)+\operatorname{deg} D
\end{aligned}
$$

Let $k$ be a natural number bigger or equal to two. Let $P_{1}, P_{2}, \ldots, P_{k}$ be $k$ different "generic" points of $X$. In our context generic means that there is a countable set of points of $X$ which have to be aroided. We choose an arbitrary numerical order of the points. This we will keep fixed. In addition we fix local coordinates $z_{l}$ around the points $P_{l}$ with $z_{l}\left(P_{l}\right)=0$ for $l=1, \ldots, k$. $X^{0}$ denotes $X \backslash\left\{P_{1}, P_{2}, \ldots, P_{k}\right\}$.

\section{The level lines}

LEMMA 1. Let $X$ be a Riemann surface of genus $g, P_{1}, P_{2}, \ldots, P_{k} k$ different points $(k \geq 2)$. Let

$$
c_{i} \in \mathbb{C} \quad, i=1, \ldots, k \text { with } \sum_{i=1}^{k} c_{i}=0
$$

then there exists a unique meromorphic differential $\rho$ on $X$ such that

(a) $\rho$ is holomorphic on $X \backslash\left\{P_{1}, P_{2}, \ldots, P_{k}\right\}$, 
(b) $\operatorname{res}_{P_{i}}(\rho)=c_{i}$, for $i=1, \ldots, k$,

(c) $\rho$ has only imaginary periods.

The proof is a generalization of the proof in [3,p.116], see also [4].

Let us apply the lemma in the following situation. Let $1 \leq l<k$ be a number, $P_{1}, \ldots, P_{l}$ and $P_{l+1}, \ldots, P_{k}$ be a partition of the points $P_{1}, P_{2}, \ldots, P_{k}$. If we set

$$
c_{i}=\frac{1}{2 l}, \quad i=1, \ldots, l \quad \text { and } \quad c_{i}=-\frac{1}{2(k-l)}, \quad i=l+1, \ldots, k
$$

we get $\sum_{i} c_{i}=0$. Let $\rho$ be the differential existing by lemma 1 . We choose $Q \in X$ with $Q \neq P_{i}, i=1, \ldots, k$.

$$
u(p):=\mathbf{R e} \int_{Q}^{P} \rho
$$

is now a harmonic function defined on $X^{0}=X \backslash\left\{P_{1}, P_{3}, \ldots, P_{k}\right\}$ with

$$
\lim _{P \rightarrow P_{l}} u(P)=-\infty, \quad i=1, \ldots, l \text { and } \lim _{P \rightarrow P_{l}} u(P)=\infty, \quad i=l+1, \ldots, k \text {. }
$$

The level line for $\tau \in \mathbb{R}$ is defined as

$$
C_{r}:=\left\{P \in X^{0} \mid u(P)=\tau\right\} \text {. }
$$

Varying $\tau$ defines a global fibration of the surface $X \backslash\left\{P_{1}, P_{2}, \ldots, P_{k}\right\}$. Each level line splits in a union of disjoint (real) curves. Singular points can only occur at the zeros of the differential $\rho$. For $\tau<0$ the level line $C_{\tau}$ splits in a collection of $l$ components $C^{r}, r=1, \ldots, l$, where each component $C^{r}$ is a curve diffeomorphic to $S^{1}$ around the point $P_{r}$. For $\tau>0$ we get the same situation around the points $P_{r},(l+1) \leq r \leq k$.

Finally, if $\omega$ is a meromorphic differential which is holomorphic on $X^{0}$, then the value of the integral of $\omega$ along any nonsingular level line will be the same.

\section{The KN - algebra and the KN - modules}

\section{DEFINITION.}

(a) The (generalized) Krichever-Novikov algebra (KN algebra) of the Riemann surface $X$ of genus $g$ and the points $(k \geq 2) P_{1}, P_{2}, \ldots, P_{k}$ is the Lie algebra of meromorphic vector fields on $X$, which are holomorphic on $X^{0}=X \backslash\left\{P_{1}, P_{2}, \ldots, P_{k}\right\}$. It is denoted by $\mathrm{KN}\left(P_{1}, P_{2}, \ldots, P_{k}\right)$ or just by $K N_{k}$.

(b) The (generalized) Krichever-Novikov module ( $\mathrm{KN}$ module) of weight $\lambda \in \mathbf{Z}$ is the vector space of meromorphic forms of weight $\lambda$ which are holomorphic on $X^{0}:=$ $X \backslash\left\{P_{1}, P_{2}, \ldots, P_{k}\right\}$. It is denoted by $F^{\lambda}\left(P_{1}, P_{2}, \ldots, P_{k}\right)$, or just by $F_{k}^{\lambda}$.

Of course, $\mathrm{KN}\left(P_{1}, P_{2}, \ldots, P_{k}\right)$ equals $F^{-1}\left(P_{1}, P_{2}, \ldots, P_{k}\right)$ as vector space. 
By taking the Lie derivative $F_{k}^{\lambda}$ is a Lie algebra module over $\mathrm{KN}_{k}$. In local terms the action can be described as follows. Let $e$ be a meromorphic vector field and $f$ be a meromorphic form of weight $\lambda$. Locally they are given as

$$
e(z)_{\mid}=\alpha(z) \frac{\partial}{\partial z}, \quad f(z)_{\mid}=\beta(z) d z^{\lambda}, \quad \text { with } d z^{\lambda}:=(d z)^{\otimes \lambda}
$$

Then the Lie derivative is given by

$$
e \cdot f(z)_{\mid}:=L_{e}(f)(z)_{\mid}=\left(\alpha(z) \frac{\partial \beta(z)}{\partial z}+\lambda \cdot \beta(z) \frac{\partial \alpha(z)}{\partial z}\right) d z^{\lambda}
$$

5. A basis for $F_{k}^{\lambda}$ in the cases $(g \geq 2, \lambda \neq 0,1)$ and $(g=0, \lambda \in \mathbf{Z})$

First we will give a set of generators which is completely symmetric in the points $P_{l}, l=1, \ldots, k$. We set

$$
M(\lambda)=(2 \lambda-1)(g-1)-1
$$

Proposition 1. Let $n_{1}, n_{3}, \ldots, n_{k-1} \in \mathbf{Z}$ and $n_{k}=M(\lambda)-\sum_{i=1}^{k-1} n_{i}$ then

$$
H^{0}\left(X, \lambda \cdot K-n_{1} P_{1}-n_{2} P_{2} \cdots-n_{k} P_{k}\right)
$$

is a one-dimensional vector space. It is generated by a (up to multiplication with a constant) unique form

$$
f=f^{\lambda}\left(n_{1}, n_{2}, \ldots, n_{k}\right) \quad \text { with } \quad \operatorname{ord}_{P_{i}}(f)=n_{i}, i=1, \ldots, k .
$$

For the details of the calculation we refer to [4]. The argument is similar to the case considered in [2]. Let me scetch the main idea. Starting point is the following

LEMMA 2. Let $\mathcal{L}$ be a line bundle, $L$ its corresponding divisor, $n$ a natural number. Set $l:=\operatorname{dim} H^{0}(X, L)$, then

$$
\operatorname{dim} H^{0}(X, L-n P)=\max (l-n, 0)
$$

if $P$ is a generic point on $X$.

We use the fact, that in our cases of $\lambda$ either

$$
\begin{gathered}
\operatorname{deg}(\lambda \cdot K)=\lambda(2 g-2) \geq 2 g-1 \quad \text { or } \\
\operatorname{deg}((1-\lambda) \cdot K)=(1-\lambda)(2 g-2) \geq 2 g-1 .
\end{gathered}
$$


Hence one of this divisors is nonspecial, which says that the second term of the left hand side of Riemann-Roch theorem vanishes [3,p.107]. With Riemann-Roch we calculate

$$
\begin{aligned}
& \operatorname{dim} H^{0}(X, \lambda \cdot K)=(2 \lambda-1)(g-1) \quad \text { or } \\
& \operatorname{dim} H^{0}(X,(1-\lambda) \cdot K)=(1-2 \lambda)(g-1) .
\end{aligned}
$$

Using (11) and assuming $P_{1}, P_{2}, \ldots, P_{k}$ to be generic we calculate

$$
\begin{aligned}
\operatorname{dim} H^{0}\left(X, \lambda \cdot K-n_{1} P_{1}-n_{2} P_{2} \cdots-n_{k} P_{k}\right) & =1, \\
\operatorname{dim} H^{0}\left(X, \lambda \cdot K-n_{1} P_{1}-n_{3} P_{2} \cdots-\left(n_{k}+a\right) P_{k}\right) & =0, a \in \mathbb{N} .
\end{aligned}
$$

The former implies the statement on the dimension, the latter implies that the generator has exactly the prescribed order at the points.

By requiring for the local representation at the point $P_{k}$

$$
f_{\mid}(z)=z_{k}^{n_{k}}\left(1+O\left(z_{k}\right)\right) d z^{\lambda}
$$

the $f=f^{\lambda}\left(n_{1}, n_{2}, \ldots, n_{k}\right)$ is completely fixed. We will assume this normalization in the following.

PROPOSITION 2. The set

$$
\left\{f^{\lambda}\left(n_{1}, n_{2}, \ldots, n_{k}\right) \mid n_{1}, n_{2}, \ldots, n_{k-1} \in \mathbf{Z}, n_{k}=M(\lambda)-\sum_{i=1}^{k-1} n_{i}\right\}
$$

is a set of generators for $F^{\lambda}\left(P_{1}, P_{2}, \ldots, P_{k}\right)$.

For $k>2$ this set is not linearly independent. To get a basis of $F_{k}^{\lambda}$ we introduce the following types of generators $(n, l \in Z)$

$$
\begin{aligned}
& f_{n}(\lambda):=f^{\lambda}(n, 0, \ldots, 0, M(\lambda)-n), \quad n \geq 0 \quad \text { type I } \\
& f_{n}(\lambda):=f^{\lambda}(n, 0, \ldots, 0, M(\lambda)-n), \quad n<0 \quad \text { type II } \\
& f_{n}^{l}(\lambda):=f^{\lambda}(0, \ldots, n, \ldots, 0, M(\lambda)-n), \quad n<0 \quad \text { type } \mathrm{III}_{l}
\end{aligned}
$$

In the definition (20) $l=2, \ldots, k-1$ and the number $n$ has to be plugged into the $l$-th position. If it is convenient we will also use the notation $f_{n}^{1}(\lambda)$ to denote generators of type I and type II. 
PROPOSITION 3. The set of

$$
f_{n}(\lambda), n \in \mathbf{Z} \text { and } f_{n}^{l}(\lambda), n \in \mathbf{Z}, n \leq-1 \text {, with } 2 \leq l \leq k-1
$$

is a basis of $F^{\lambda}\left(P_{1}, P_{2}, \ldots, P_{k}\right)$.

If there is no ambiguity we will drop the $\lambda$ in the notation. Due to the special importance of certain weights we introduce

$$
e_{n}^{l}:=f_{n}^{l}(-1), \quad \text { and } \quad \Omega_{n}^{l}:=f_{n}^{l}(2)
$$

and (assuming the result of the next section already)

$$
A_{n}^{l}:=f_{n}^{l}(0), \quad \text { and } \quad \omega_{n}^{l}:=f_{n}^{l}(1)
$$

Of course, it is also possible to embed the Riemann surface into its Jacobian and to describe in some sense more explicitly the above forms in terms of theta functions, prime forms etc. as it was done for $k=2$ in [11] and [14]. This will be covered in [4].

6. A basis for $F_{k}^{\lambda}$ in the cases $(g \geq 2, \lambda=0$ or $\lambda=1)$ and $(g=1, \lambda \in Z)$

Due to the fact that $\lambda \cdot K$ is a special divisor we have to modify the argument and the basis.

Proposition 4. (g $\geq 2, \lambda=1)$. A set of symmetric generators of $F_{k}^{1}$ is given by $\left(n_{i} \in \mathbf{Z}, i=1, \ldots, k-1\right)$

$$
\begin{gathered}
f^{1}\left(n_{1}, n_{2}, \ldots, n_{k-1},(g-1)-\sum_{i=1}^{k-1} n_{i}\right), \quad n_{i} \geq 0, \sum_{i=1}^{k-1} n_{i} \leq(g-1), \\
f^{1}\left(n_{1}, n_{2}, \ldots, n_{k-1},(g-2)-\sum_{i=1}^{k-1} n_{i}\right), \quad n_{i} \neq-1,
\end{gathered}
$$

where at least one $n_{i} \leq-2$ or $\sum_{i=1}^{k-1} n_{i} \geq g$ and

$$
\begin{aligned}
& f^{1}(-1,-1,0, \ldots, 0), f^{1}(-1,0,-1, \ldots, 0), \ldots, \\
& f^{1}(0,-1,-1, \ldots, 0), \ldots, f^{1}(0, \ldots, 0,-1,-1) .
\end{aligned}
$$

The elements $f$ in case (22) and (23) are uniquely given by a similar argument as above. In case (24) we understand by $f^{1}(0,0, \ldots, 0,-1, \ldots,-1, \ldots, 0)$ the unique differential given by lemma 1 with $c_{l}=-1$ and $c_{m}=1$. Here $l$ and $m$ are the entries being equal to -1 in the description of $f$ with $l<m$. Here again we have similar types $(n \in Z)$

$$
\omega_{n}:=f_{n}(1):=f^{1}\left(n, 0, \ldots, 0,\left\{\begin{array}{l}
g-2-n, n \geq g \\
g-1-n, 0 \leq n \leq g-1
\end{array}\right), \quad\right. \text { type I }
$$




$$
\begin{aligned}
& \omega_{n}:=f_{n}(1):=\left\{\begin{array}{ll}
f^{1}(-1,0, \ldots, 0,-1), & n=-1 \\
f^{1}(n, 0, \ldots, 0, g-2-n), & n \leq-2
\end{array} \quad\right. \text { type II } \\
& \omega_{n}^{l}:=f_{n}^{l}(1):=\left\{\begin{array}{ll}
f^{1}(0, \ldots,-1, \ldots, 0,-1), & n=-1 \\
f^{1}(0, \ldots, n, \ldots, 0, g-2-n), & n \leq-2
\end{array} \quad \text { type III }{ }_{l}\right.
\end{aligned}
$$

In (27) we have $l=2, \ldots, k-1$.

PROPOSITION 5. The set of the generators (25)-(27) is a basis for $F^{-1}\left(P_{1}, P_{2}, \ldots, P_{k}\right)$.

In the case of genus $1 K$ is the trivial bundle. Hence all tensor powers are again trivial. For this reason a basis of $F_{k}^{0}$ is a basis for all $F_{k}^{\lambda}$.

Proposition 6. $(g \geq 2, \lambda=0$ or $g=1, \lambda \in \mathbf{Z})$. A set of symmetric generators is given by $\left(n_{i} \in \mathbf{Z}, i=1, \ldots, k-1\right)$

$$
\begin{gathered}
f^{0}\left(n_{1}, n_{2}, \ldots, n_{k-1},-g-\sum_{i=1}^{k-1} n_{i}\right), \text { at least one } n_{i}>0, \text { or } \sum_{i=1}^{k-1} n_{i}<-g, \\
f^{0}\left(n_{1}, n_{3}, \ldots, n_{k-1},-g-1-\sum_{i=1}^{k-1} n_{i}\right), n_{i} \leq 0, \sum_{i=1}^{k-1} n_{i} \geq-g-1, \\
f^{0}(0,0,0, \ldots, 0) \equiv 1
\end{gathered}
$$

In case (28) uniqueness is again by a similar argument as above. In case (29) such a $f$ is only unique up to muliplication with a constant and adding a constant. Hence normalization at the point $P_{k}$ does not fix it completely. We will give a method for fixing it by duality later on. The types are $(n \in Z)$

(31) $A_{n}:=f_{n}(0):=\left\{\begin{array}{ll}f^{0}(n, 0, \ldots, 0,-g-n), & n>0 \\ f^{0}(0,0,0, \ldots, 0) \equiv 1, & n=0\end{array} \quad\right.$ type I

(32) $A_{n}:=f_{n}(0):=\left\{\begin{array}{ll}f^{0}(n, 0, \ldots, 0,-g-1-n), & -g \leq n<0 \\ f^{0}(n, 0, \ldots, 0,-g-n), & n \leq-(g+1)\end{array} \quad\right.$ type II

(33) $A_{n}^{l}:=f_{n}^{l}(0):=\left\{\begin{array}{ll}f^{0}(0, \ldots, n, \ldots, 0,-g-1-n), & -g \leq n<0 \\ f^{0}(0, \ldots, n, \ldots, 0,-g-n), & n \leq-(g+1)\end{array}\right.$ type III with $l=2, \ldots, k-1$.

PROPOSITION 7. The set of the generators (31)-(33) is a basis for $F^{0}\left(P_{1}, P_{2}, \ldots, P_{k}\right)$. 


\section{The structure constants}

If we choose the basis in $\mathrm{KN}\left(P_{1}, P_{2}, \ldots, P_{k}\right)$ and the basis in $F^{\lambda}\left(P_{1}, P_{2}, \ldots, P_{k}\right)$ as above then the module structure of $F_{k}^{\lambda}$ over $\mathrm{KN}_{k}$, resp. the structure of the algebra $\mathrm{KN}_{k}$ itself for $\lambda=-1$, is completely fixed by the structure constants $C_{\alpha, \beta}^{\gamma} \in \mathbb{C}$, given by

$$
e_{\alpha} \cdot f_{\beta}=\sum_{\gamma}^{\prime} C_{\alpha, \beta}^{\gamma} f_{\gamma}
$$

Here $\alpha, \beta, \gamma$ are generalized indices. The structure constants are depending on the weight $\lambda \cdot \Sigma^{\prime}$ denotes that only finitely many $f_{\gamma}$ actually occur.

By doing local calculations at the points $P_{1}, P_{2}, \ldots, P_{k}$ (similar to $[3, p .115]$ ) we can determine which indices $\gamma$ occur. We restrict ourselves to $g \geq 2$ and $\lambda \neq 0,1$ or $g=0$ and $\lambda \in \mathbf{Z}$. In the remaining cases there are some minor modifications due to the exceptional elements in the basis (see [4]).

Let us fix $\lambda$. Depending on the type of the basis elements we get the following result: (type I, type I)

$$
e_{n} \cdot f_{m}=\sum_{r=\max (0, n+m-1)}^{n+m-1+3 g} A_{n, m}^{r}(\lambda) f_{r}
$$

(type I,type II) and vice versa

$$
e_{n} \cdot f_{m}=\sum_{r=0}^{n+m-1+a g} A_{n, m}^{r}(\lambda) f_{r}+\sum_{r=n+m-1}^{\min (-1, n+m-1+3 g)} A_{n, m}^{r}(\lambda) f_{r}^{1} .
$$

Of course one of the sum could be the empty sum. This coefficients are exactly the coefficients of the KN algebra (modules) of the two points $P_{1}$ and $P_{k}$. In the boundary cases we get

$$
A_{n, m}^{n+m-1+\partial g}(\lambda)=-(m+\lambda n)-g(1+\lambda), \quad A_{n, m}^{n+m-1}(\lambda)=(m+\lambda n) \frac{a_{n} b_{m}}{b_{n+m-1}}
$$

Here $a_{n}$ is the leading coefficient of $e_{n}$ at the point $P_{1}$ and $b_{m}$ the leading coefficient of $f_{m}$ at the point $P_{1}$.

(type $\mathrm{I}$,type $\mathrm{III}_{l}$ ) and vice versa

$$
e_{n} \cdot f_{m}^{l}=\sum_{r=0}^{n+m-1+3 g} A_{n, m}^{r, l}(\lambda) f_{r}+\sum_{r=m-1}^{\min (-1, n+m-1+2 g)} A_{n, m}^{r, l}(\lambda) f_{r}^{l}
$$




$$
e_{n}^{l} \cdot f_{m}=\sum_{r=0}^{n+m-1+3 g} B_{n, m}^{r, l}(\lambda) f_{r}+\sum_{r=n-1}^{\min (-1, n+m-1+3 g)} B_{n, m}^{r, l}(\lambda) f_{r}^{l}
$$

The basic method for calculation of the coefficient, is to write $e_{n}$, resp. $f_{m}$ as linear combination of the basis of the $\mathrm{KN}$ algebra $\mathrm{KN}\left(P_{l}, P_{k}\right)$, resp. $\mathrm{KN}$ module, do the calculation as above and transfer the result back in terms of our basis. For a detailed calculation see [4]. As can be seen in (38) there is always a term $f_{-n-1}^{l}$ even if $m$ was chosen to be a big positive number.

(type $\mathrm{III}_{l}$, type $\mathrm{III}_{l}$ )

$$
e_{n}^{l} \cdot f_{m}^{l}=\sum_{r=0}^{n+m-1+3 g} C_{n, m}^{r, l}(\lambda) f_{r}+\sum_{r=n+m-1}^{\min (-1, n+m-1+3 g)} C_{n, m}^{r, l}(\lambda) f_{r}^{l} .
$$

(type $\mathrm{III}_{l}$, type $\left.\mathrm{III}_{h}\right), h \neq l$ or (type II, type $\left.\mathrm{III}_{l}\right)$ :

$$
e_{n}^{l} \cdot f_{m}^{h}=\sum_{r=n-1}^{-1} D_{n, m}^{r, l, h}(\lambda) f_{r}^{l}+\sum_{r=m-1}^{-1} E_{n, m}^{r, l, h}(\lambda) f_{r}^{h}+\sum_{r=0}^{n+m-1+3 g} F_{n, m}^{r, l, h}(\lambda) f_{r} .
$$

If $n+m \leq-3 g$ the last sum will not appear. Nevertheless in the first and second sum all terms will occur up to $r=-1$.

For $k=2$ this is exactly the result of Krichever and Novikov [1]. In their rule of indexing the above looks like

$$
e_{(i)} \cdot f_{(j)}=\sum_{l=-g_{0}}^{g_{0}} R_{i, j}^{l} f_{(i+j+l)}, \quad g_{0}=\frac{3}{2} g
$$

Both rules of indexing are related by

$$
i=n+\frac{3}{2} g-1 \quad \text { and } \quad j=m+\frac{g}{2}-\lambda(g-1) .
$$

Here the structure looks very symmetric. This symmetry does not occur for $k>2$. Hence we decided not to choose this rule of indexing.

\section{Example}

To illustrate the result we calculate in the case of $X=\mathbb{P}^{1}$ (hence $g=0$ ) the algebra $\mathrm{KN}\left(P_{1}, P_{2}, \ldots, P_{k}\right)$. We choose such a parametrization $z$ of $X$ that $P_{1}$ corresponds 
to $z=0$ and $P_{k}$ corresponds to $z=\infty$. The points $P_{l}$ correspond to $a_{l} \in \mathbb{C}$ for $l=2, \ldots, k-1$. In this parametrization it is easy to give the basis in an explicit way

$$
\begin{aligned}
& e_{n}=z^{n} \frac{\partial}{\partial z}, \quad n \geq 0 \quad \text { type I } \\
& e_{n}=z^{n} \frac{\partial}{\partial z}, \quad n<0 \quad \text { type II } \\
& e_{n}^{\prime}=\left(z-a_{l}\right)^{n} \frac{\partial}{\partial z}, \quad n<0 \quad \text { type III } .
\end{aligned}
$$

Here we normalized (contrary to the general rule) in such a way that the leading coefficient at $\infty$ is equal to -1 .

By direct calculation we see:

$$
\begin{aligned}
& {\left[e_{n}, e_{m}\right]=(m-n) e_{n+m-1}, \quad n, m \in \mathbf{Z}} \\
& {\left[e_{n}, e_{m}^{l}\right]=\sum_{r=0}^{m+n-1} A_{n, m}^{r, l} e_{r}+\sum_{r=m-1}^{\min (-1, m+n-1)} A_{n, m}^{r, l} e_{r}^{l} \quad n \geq 0}
\end{aligned}
$$

with $l=2, \ldots, k-1$ and

(46) $A_{n, m}^{r, l}=\left(\sum_{t=r}^{m+n-1}(-1)^{t+r}\left(\begin{array}{l}t \\ r\end{array}\right)\left(\begin{array}{c}n \\ t-m+1\end{array}\right)(2 m-1-t)\right) a_{l}^{n+m-1-r}, \quad r \geq 0$

$(4 \mathbf{b a}) A_{n, m}^{r, l}=\left(\begin{array}{c}n \\ r-m+1\end{array}\right) \cdot a_{l}^{n+m-1-r} \cdot(2 m-1-r), \quad r<0$

Furthermore for $l=2, \ldots, k-1$

$$
\left[e_{n}^{l}, e_{m}^{l}\right]=(m-n) e_{n+m-1}^{l}, \quad n, m<0 .
$$

In the remaining case $1 \leq r, l \leq k-1, r \neq l$ we get

$$
\left[e_{n}^{r}, e_{m}^{l}\right]=\sum_{s=n-1}^{-1} D_{n, m}^{s, r, l} e_{s}^{r}+\sum_{s=m-1}^{-1} E_{n, m}^{s, r, l} e_{s}^{l}
$$

with (setting $q=m+n-1$ and $a_{1}=0$ )

$$
\begin{aligned}
& D_{n, m}^{s, r, l}=\left(\begin{array}{c}
m \\
-n+1
\end{array}\right)\left(a_{r}-a_{l}\right)^{q-\theta}(\varepsilon-2 n+1) \\
& E_{n, m}^{s, r, l}=-D_{m, n}^{s, l, r} .
\end{aligned}
$$

In particular, we see that $e_{-1}^{r}$ and $e_{-1}^{l}$ will always occur. 


\section{Duality}

If we multiply a form of weight $\lambda$ and a form of weight $1-\lambda$ we get a form of weight 1 , hence a 1-differential. By taking the integral over suitable curves we get a kind of a duality pairing between the above forms. We choose a differential (see equation (26))

$$
\rho=\frac{1}{k-1} \sum_{l=1}^{k-1} \omega_{-1}^{l} .
$$

It has residue $-1 /(k-1)$ at the points $P_{1}, \ldots, P_{k-1}$ and residue +1 at the point $P_{k}$. The level line is a circle around $P_{k}$ for $r \ll 0$ and a collection of circles around the other points for $\tau>0$. Let us denote such a circle (with orientation) around $P_{l}$ by $C^{l}$. For smooth $C_{\tau}$ and for $\omega \in F^{1}\left(P_{1}, P_{2}, \ldots, P_{k}\right)$ we get

$$
\sum_{l=1}^{k-1} \frac{1}{2 \mathbf{i} \pi} \oint_{C^{l}} \omega=\frac{1}{2 \mathbf{i} \pi} \oint_{C_{r}} \omega=\frac{1}{2 \mathbf{i} \pi} \oint_{C^{*}} \omega .
$$

Of course, we could have chosen arbitrary circles $C^{l}$ around the points $P_{l}$ without changing the value of the integral.

PROPOSITION 8. Let $v \in F^{1-\lambda}\left(P_{1}, P_{2}, \ldots, P_{k}\right)$ have the representation

$$
\begin{gathered}
v=\sum_{n \geq 0}^{\prime} r_{n} f_{n}(1-\lambda)+\sum_{n<0}^{\prime} r_{n} f_{n}(1-\lambda)+ \\
\sum_{i=2}^{k-1} \sum_{n<0}^{\prime} e_{n}^{l} f_{n}^{l}(1-\lambda) .
\end{gathered}
$$

Then the coefficients can be calculated by

$$
\begin{aligned}
r_{n} & =\frac{1}{2 \mathbf{i} \pi} \oint_{C^{ \pm}} f_{-n-1}(\lambda) \cdot v=\frac{1}{2 \mathbf{i} \pi} \oint_{C_{r}} f_{-n-1}(\lambda) \cdot v, \quad n \geq 0 \\
r_{n} & =\frac{1}{2 \mathbf{i} \pi} \oint_{C^{1}} f_{-n-1}(\lambda) \cdot v \quad n<0 \\
\boldsymbol{s}_{-1}^{l} & =\frac{1}{2 \mathbf{i} \pi} \oint_{C^{l}} f_{0}(\lambda) \cdot v \\
\boldsymbol{s}_{-2}^{l} & =\frac{1}{2 \mathbf{i} \pi} \oint_{C^{l}} f_{1}(\lambda) \cdot v-s_{-1}^{l} \alpha_{1,-1}^{l}(\lambda) \\
\quad & \\
\boldsymbol{s}_{-r}^{l} & =\frac{1}{2 \mathbf{i} \pi} \oint_{C^{l}} f_{r-1}(\lambda) \cdot v-\sum_{p=1}^{r-1} s_{-p}^{l} \alpha_{r-1,-p}^{l}(\lambda) .
\end{aligned}
$$


Here it is defined $(r \geq 0, t<0)$

$$
\alpha_{r, k}^{\prime}(\lambda)=\frac{1}{2 \mathbf{i} \pi} \oint_{C^{t}} f_{r}(\lambda) \cdot f_{t}^{\prime}(1-\lambda)
$$

We get

$$
\alpha_{n,-n-1}^{l}(\lambda)=1 \text { and } \alpha_{n, m}^{l}(\lambda)=0 \text { if } m<-n-1 \text {. }
$$

In the case of $k=2$ there are no terms of the third kind. Hence we get the (well known) result

$$
\frac{1}{2 \mathbf{i} \pi} \oint_{C_{+}} f_{n}(\lambda) \cdot f_{m}(1-\lambda)= \begin{cases}1 & m=-n-1 \\ 0 & m \neq-n-1\end{cases}
$$

In case of genus $g$ equal to 1 or in case of $g \geq 2$ and $\lambda=0$ or 1 we have to add certain constants to the elements $A_{n}^{l}$ for $-g \leq n \leq-1$ (33) to make the proposition 8 also true in this case. But remember, up to now we were only able to fix exactly this elements up to an addition of a constant (see proposition 6). With the duality requirement they are completely determined. See [4] for details.

\section{The central extension}

Starting from our Krichever-Novikov algebra $\mathrm{KN}=\mathrm{KN}\left(P_{1}, P_{2}, \ldots, P_{k}\right)$ we consider central extensions $\widehat{\mathrm{KN}}$ of it. Let $E_{n}^{l}$ denote a fixed lift to $\widehat{\mathrm{KN}}$ of the basis element $e_{n}^{l}$ of $\mathrm{KN}$. Then $\widehat{\mathrm{KN}}$ is generated by a central element $t$ and the set of $E_{n}^{l}$ ( $n$ and $l$ as in equation (21)). We get

$$
\begin{gathered}
{\left[E_{\alpha}, t\right]=0} \\
{\left[E_{\alpha}, E_{\beta}\right]=\sum_{\gamma} C_{\alpha, \beta}^{\gamma} E_{\gamma}+\chi\left(e_{\alpha}, e_{\beta}\right) t}
\end{gathered}
$$

Here $\alpha, \beta, \gamma$ are generalized indices (i.e. $E_{\alpha}=E_{n}^{l}$ ), $C_{\alpha, \beta}^{\gamma}$ are the structure constants of the algebra $\mathrm{KN}$

$$
\left[e_{\alpha}, e_{\beta}\right]=\sum_{\gamma} C_{\alpha, \beta}^{\gamma} e_{\gamma}
$$

and $\chi(\alpha, \beta) \in \mathbb{C}$ is a 2-cocycle. It is defined for every pair of vector fields and it fulfills

$$
\begin{gathered}
\chi(\alpha, \beta)=-\chi(\beta, \alpha) \\
\chi([f, g], h)+\chi([g, h], f)+\chi([h, f], g)=0 .
\end{gathered}
$$

These conditions are sufficient and necessary for $\widehat{\mathrm{KN}}$ being a Lie algebra.

To construct central extensions we use the method of [2]. 
DEFINITION. Let $\left(U_{\alpha}, z_{\alpha}\right)$ be a covering of $X$ by coordinate patches and let $z_{\beta}=f_{\alpha \beta}\left(z_{\alpha}\right)$ be the transition functions for non-empty $U_{\alpha} \cap U_{\beta}$. A meromorphic projective connection is a collection of local meromorphic functions $R_{\alpha}\left(z_{\alpha}\right)$ which are related on nonempty $U_{a} \cap U_{\beta}$ by

$$
R_{\beta}\left(z_{\beta}\right)\left(\frac{\partial z_{\beta}}{\partial z_{\alpha}}\right)^{2}=R_{\alpha}\left(z_{\alpha}\right)+S\left(f_{\alpha \beta}\right) .
$$

Here $S(h)$ is the Schwartzian derivative. It is defined as

$$
S(h)=\frac{h^{\prime \prime \prime}}{h^{\prime}}-\frac{3}{2}\left(\frac{h^{\prime \prime}}{h^{\prime}}\right)^{2} .
$$

The' denotes derivation with respect to the local variable $z$.

If all local functions are holomorphic we call $R$ a holomorphic projective connection. There exists always a holomorphic projective connection [18,p.202]. Due to the transformation law (65) the difference of two projective connections is always a quadratic differential (i.e. a form of weight 2). Hence by fixing one holomorphic connection $R_{0}$ we can get all of them by adding forms of weight 2 . In the following we are mainly interested in meromorphic ones which are holomorphic on $X \backslash\left\{P_{1}, P_{2}, \ldots, P_{k}\right\}$ and have a pole of at most order 1 at the points $P_{l}, l=1, \ldots, k$. We get for $g \geq 2$

$$
R=R_{0}+\sum_{n=0}^{3 g-3} c_{n} \Omega_{n}+\sum_{l=1}^{k-1} c_{-1}^{l} \Omega_{-1}^{l}, \quad c_{n}, c_{-1}^{l} \in \mathbb{C}
$$

resp. for $g=1\left(\Omega_{n}^{l}=A_{n}^{l}\right)$

$$
R=R_{0}+c_{0} \Omega_{0}+\sum_{l=1}^{k-1} c_{-1}^{l} \Omega_{-1}^{l}, \quad c_{0}, c_{-1}^{l} \in \mathbb{C}
$$

For $g=0$ there is only something to add to $R_{0}$ if $k \geq 4$

$$
R=R_{0}+\sum_{b=1}^{k-8} c_{s} \Omega^{\circ}, \quad c_{\theta} \in \mathbb{C} .
$$

The $\Omega^{\circ}$ are certain forms of weight 2 . If we use "standard coordinates" for $g=1$ and $g=0$ we can use $R_{0} \equiv 0$ in these cases.

With the help of these projective connections we set for vector fields $e, h$ with the local representations

$$
e_{1}=f(z) \frac{\partial}{\partial z}, \quad h_{1}=g(z) \frac{\partial}{\partial z}
$$




$$
\bar{\chi}(e, h):=\left(\frac{1}{2}\left(f^{\prime \prime \prime} g-f g^{\prime \prime \prime}\right)-R \cdot\left(f^{\prime} g-f g^{\prime}\right)\right) d z .
$$

This is a meromorphic 1 -form which we can integrate along the level lines $C_{r}$ according to the differential $\rho$ of equation (51). We set with $\hat{c} \in \mathbb{C}$ an arbitrary constant

$$
\chi(e, h)=\frac{\hat{c}}{24 \pi \mathbf{i}} \oint_{C_{+}} \bar{\chi}(e, h)=\frac{\hat{c}}{24 \pi \mathbf{i}} \oint_{C^{\star}} \bar{\chi}(e, h) .
$$

PROPOSITION 9. $\chi(e, h)$ defines a 2-cocycle, hence a central extension of the $K N$ algebra.

\section{Semiinfinite wedge representations}

We $f x$ a weight $\lambda$. Let $F$ be $F^{\lambda}\left(P_{1}, P_{2}, \ldots, P_{k}\right), f_{n}^{l}$ the basis of $F, e_{n}^{l}$ the basis of $\mathrm{KN}\left(P_{1}, P_{3}, \ldots, P_{k}\right)$. We want to give the elements of the basis a $\mathbf{Z}$ - graduation

$$
\begin{array}{ll}
f_{(i)}:=f_{n}, & n \geq 0 \\
f_{(i)}:=f_{n}^{l}, & n<0, l=1, \ldots, k-1 \text { where } i=(n+1)(k-1)-l .
\end{array}
$$

A semiinfinite form is an element of the vector space

$$
H=H^{\lambda}\left(P_{1}, P_{3}, \ldots, P_{k}\right)
$$

generated by the formal elements

$$
w=f_{\left(i_{r}\right)} \wedge f_{\left(i_{r+1}\right)} \wedge \ldots f_{\left(i_{0}\right)} \wedge f_{(m)} \wedge f_{(m+1)} \wedge \ldots
$$

with

$$
i_{r}<i_{r+1}<\ldots<i_{0}<m \text {. }
$$

The dots in the right part of (75) means that starting from an arbitrary index $m$ all elements with index $k \geq m$ appear.

We have

$$
e_{(i)} \cdot f_{(j)}=\sum_{(l)}^{\prime} G_{i, j}^{l} f_{(l)}, \quad G_{i, j}^{l} \in \mathbb{C}
$$

This action of $\mathrm{KN}$ on $F$ we want to transfer onto the vector space $H$. We try the following naive definition (Leibniz rule)

$$
\begin{aligned}
& e . w:=\left(e \cdot f_{\left(i_{1}\right)}\right) \wedge f_{\left(i_{2}\right)} \wedge \ldots+f_{\left(i_{1}\right)} \wedge\left(e \cdot f_{\left(i_{2}\right)}\right) \wedge \ldots+\ldots \\
&+f_{\left(i_{1}\right)} \wedge \ldots \wedge\left(e \cdot f_{(m)}\right) \wedge f_{(m+1)} \ldots+\ldots+\ldots
\end{aligned}
$$


The $\wedge$ indicates how to calculate the result. The rules are $(\Phi, \Psi$ and $v$ are neighbour pieces)

$$
\begin{aligned}
& \Phi \wedge f_{j} \wedge \Psi \wedge f_{i} \wedge v:=-\Phi \wedge f_{i} \wedge \Psi \wedge f_{j} \wedge v, \quad j>i \\
& \Phi \wedge f_{i} \wedge \Psi \wedge f_{i} \wedge v:=0 \\
& \Phi \wedge\left(\sum_{i=1}^{r} c_{i} f_{i}\right) \wedge v:=\sum_{i=1}^{r} c_{i}\left(\Phi \wedge f_{i} \wedge v\right) .
\end{aligned}
$$

The definition (78) makes sense if there are only finitely many terms on the right hand side. A closer examination [4] shows that this only works for subalgebras of $\mathrm{KN}$. We introduce the following subalgebras

$$
\begin{aligned}
& \mathrm{KN}_{+}:=\left\langle e_{n} \mid n \geq 2\right\rangle \\
& \mathrm{KN}_{-}^{l}:=\left\langle e_{n}^{l} \mid n \leq-3 g\right\rangle \quad l=1, \ldots, k-1
\end{aligned}
$$

For the elements of $\mathrm{KN}_{+}$and of $\mathrm{KN}_{-}^{l}$ the action $(78)$ is well defined. Hence $H$ is a module over these algebras. Unfortunately this is not true for $\mathrm{KN}_{-}^{l}, l \geq 2$.

In the case of $\mathrm{KN}_{2} H$ becomes a module over a central extension of $\mathrm{KN}$ [1]. It is reasonable to expect that this will be true in the general situation. This question and a closer study of the central extensions of $\mathrm{KN}_{k}$ is under investigation [5][1].

\section{Acknowledgements}

I like to thank J.Wess who got me interested in the Krichever-Novikov algebra. I also like to thank L.Bonara, R.Dick, M.Matone and R. Weissauer for discussions on the subject. R.Dick informed me after completion of this work that he also got some results in this direction. 


\section{References}

1. I.M. Krichever and S.P. Novikov, Algebras of Virasoro Type, Riemann Surfaces and Structures of the Theory of Solitone, Funk. Anal. i. Pril. 21 (2) (1987), 46.

2. I.M. Krichever and S.P. Novikov, Virasoro Type Algebras, Riemann Surfaces and Strings in Minkowski Space, Funk. Anal. i. Pril. 21 (4) (1987), 47.

3. M. Schlichenmaier, "An Introduction to Riemann Surfaces, Algebraic Curves and Moduli Spaces", Lecture Notes in Physics Vol. 322, Springer, 1989.

4. M. Schlichenmaier, Verallgemeinerte Krichever-Novikov Algebren und deren Darstellungen, (to appear).

5. M. Schlichenmaier, Central Extensions and Semiinfinite Wedge Representations of Generalized Krichever-Novikov Algebras, (in preparation).

6. L. Bonora, M. Bregola P. Cotta-Ramusino and M. Martinelli, Virasoro-Type Algebras and BRST operators on Riemann Surfaces, Phys. Lett. B205 (1988), 53-56.

7. L. Bonora, M. Martinelli, M. Rinaldi and J.Russo, Neuveu-Schwarz- and RamondType Superalgebras on Genus g Riemann Surfaces, Phys. Lett. B206 (1988), 444-450.

8. L. Bonora, M. Rinaldi, J. Russo and K. Wu, The Sugawara Construction on Genus $g$ Riemann Surfaces, Phys. Lett. B208 (1988), 440-446.

9. L. Bonora, M. Matone and M. Rinaldi, Relation between Representations of $K N$ and Virasoro Algebras, Phys. Lett. B216 (1989), 313-319.

10. L. Bonora, M. Martinelli, M. Rinaldi and K.Wu, Conformal Invariance on a Generic Riemann Surface in the Presence of a Non-flat Background Metric, CERN-TH.5228/88 and SISSA 135/88/EP.

11. L. Bonora, A. Lugo, M. Matone and J. Russo, A Global Operator Formalism on Higher Genws Riemann Surfaces: b-c Systems, SISSA 67/88/EP to appear in Comm. Math. Phys..

12. P. Cotta-Ramusino, M. Martinelli and M. Mintchev, Deformations of Complex Structures and Representations of Krichever-Novikov Algebrae, CERN.TH.5181/88.

13. J. Alberty, A. Taormina and P. van Baal, Relating Kac-Moody, Virasoro and Krichever-Novikov Algebras, Comm. Math. Phys. 120 (1988), 249-260.

14. A. Lugo and J. Russo, Hamiltonian Formulation and Scattering A mplitudes in String Theory at Genue $g$, SISSA 83/88/EP.

15. N.S. Hawley and M. Schiffer, Half Order Diferentiale on Riemann Surfaces, Acta Math. 115 (1966), 199-236. 